\title{
No conclusive evidence favouring cast over direct post and core preparations
}

\author{
Are cast posts more effective than direct posts when patients require \\ post-and-core restorations?
}

\begin{abstract}
Heydecke G, Peters MC. The restoration of endodontically treated, single rooted teeth with cast or direct posts and cores: a systematic review. J Prosthet Dent 2002; 87:380-386
\end{abstract}

Data sources English, French or German articles were identified in Medline and EMbase, and in the reference lists of retrieved articles.

Study selection The in-vitro studies were of single rooted teeth (no resin analogues) and load angles of 130-135. In-vivo studies were of $\geqslant 3$ years' duration. These included teeth that could be identified separately with complete crown restoration including fixed partial denture abutments and detectable information regarding success or failure.

Data extraction and synthesis For in-vitro studies, primary outcome was load-to-failure. For in-vivo studies, failure was defined as a need for recementing, a new restoration of any kind, or tooth extraction. A qualitative synthesis of all included in-vitro and in-vivo studies was performed along with a meta-analysis of four in-vitro studies.

Results Ten in-vitro and six in-vivo studies were included. The metaanalysis of four in-vitro studies revealed no significant difference in fracture load. There was also little difference in the mode of fracture across the 10 in-vitro studies. For three of the in-vivo studies it was possible to construct a life table that indicated the survival rate for cast posts was between 87 and $88 \%$ and for direct posts was $86 \%$ at 72 months.

Conclusions There is no conclusive evidence favouring cast over direct post and core preparations or vice versa. The literature on the clinical success of post-retained cores is scare and randomised controlled trials are needed.

\begin{abstract}
Commentary
The post-and-core restoration has challenged dental clinicians for a long time. Although many different forms and procedures have been advocated over the past few decades, the cast post-and-core remained the gold standard for building up severely damaged teeth. Moreover, it was thought that these restorations could strengthen weakened teeth and as a result they were promoted to prevent fracture of the endodontically treated tooth. When, in the mid1980s, it was demonstrated posts might weaken the root, especially if sound tooth tissue was removed to allow for indirect prosthodontic procedures, questions were raised about the advisability of using posts. At that time direct post-and-core systems were also introduced. These offered the possibility of using posts without the necessity of removing undercuts, since composite material is used for the core. In this sense this technique could be considered toothtissue-saving. The present study is an attempt to organise, in a systematic review, the results that have been achieved using direct and cast post-and-core restorations, as presented in the abundant literature on this topic.
\end{abstract}

Address for correspondence: Dr Guido Heydecke, Department of Prosthodontics, Schoo of Dentistry, Albert-Ludwigs University, Hugstetter Strasse 55, 79106 Frieburg, Germany. E-mail: heydecke@umich.edu
The paper presents a useful overview on post-and-core restorations for single-rooted teeth, but the level of the evidence provided is low. Although the title of the paper promises a systematic review, the study does not meet the criteria allowing it to be considered as such. ${ }^{1}$ The most systematic part in this review is the description of the literature search procedure. The search procedure, however, is not verifiable: only inclusion criteria are presented, so there is no information about the excluded papers and the reasons why they were excluded (exclusion criteria). Moreover, the reliability of the procedure is not demonstrated, there is no indication of how many observers were involved in the selection, how examiners were calibrated, and what their level of agreement was. Other points of concern are, first, that no year limits were presented (only the limits for reference list search); second, that it is unclear how many papers were retrieved directly by the use of the provided keywords; and, third, the number of papers identified in the reference lists of retrieved papers and then included indirectly is not reported. The latter is important because the outcome can be highly biased by selective citations that might support the opinions of the primary authors. In conclusion, the selection procedure is not transparent and not reproducible on the basis of the description given and might lead to completely different results when followed by others.

The present review is an update of an earlier meta-analysis of clinical studies of post-and-core restorations, ${ }^{2}$ with the emphasis on the comparison of classic cast post-and-cores with direct post-andcores. In addition, the authors attempt to combine and analyse results of in-vitro studies. In respect of the first aim, it is disappointing to learn that no useful clinical data appeared in the dental literature after the publication of the 1993 review. $^{2}$ As a result both reviews deal with the same set of publications. A recently published systematic review ${ }^{3}$ showed that results of systematic reviews on related prosthodontic subjects might be similar even if different processes are followed or different sets of studies are involved. It was suggested that if no strict rules are followed for reviewing, or when observational studies are involved in the process, validation of the outcomes is only possible by independently repeated analyses. It is interesting to discover whether the present review verifies the previously-published evidence: comparison of both reviews shows that the results are slightly different. It appears that the endpoints of cast posts' survival in primary studies in the present review are within the confidence levels of those of the earlier one. This could mean that the primary data were interpreted approximately the same. A closer look, however, shows that the present review considers only anterior restorations whereas the earlier publication also included posterior ones. Therefore it cannot be stated that the present review validates the previous analysis.

One comment should be added to the analysis of the in-vivo studies. Since it appeared to be impossible to aggregate the results into one overall survival rate, separate survival curves of the results of the useful primary studies were presented in a single figure (see Figure 1). Although such presentation may be convenient for the reader, it should not be used for direct comparison because comparing outcomes of different observational studies cannot 


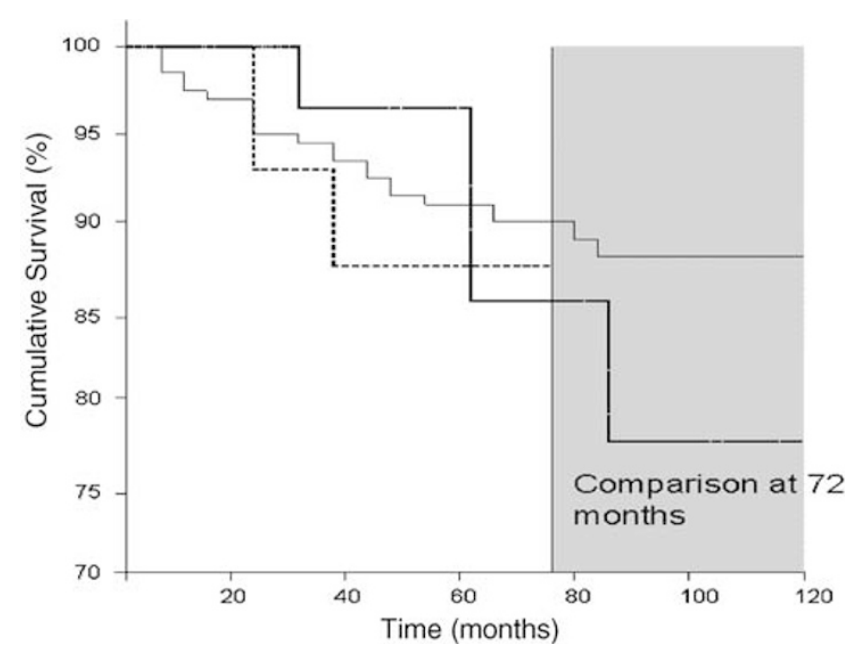

Figure 1. Slightly amended version of Figure 2 from the original paper ${ }^{4}$ showing survival rates for post-and-cores for anterior teeth in three studies provided for comparison. This comparison of observational studies is inappropriate, however, because the conditions of the studies may differ significantly.

demonstrate that the efficacy of one therapy is better than that of another one.

With respect to the review of in-vitro studies, it appeared that four studies were useful for aggregation of failure load data. The paper suggests that the homogeneity of conditions was sufficient to allow secondary analysis. The variation between the primary results (the lowest mean failure load for direct posts-and-cores was $425 \mathrm{~N}$ : the highest was $1053 \mathrm{~N}$ ) was such that the homogeneity claimed is doubtful. Although it might be true that there is no statistical difference between the failure load resistances of cast and direct post-and-core restorations - as was reported in this paper - the lack of power of the meta-analysis might also be responsible for this finding. In this respect it is surprising to see that the weighed means in the meta-analysis as presented in this paper show relative small confidence intervals, in contrast with the wide variation seen in the primary data. On the basis of the few data used for comparison it is therefore not justifiable to conclude that cast post-and-cores provide equal failure load resistance as direct post-and-cores do.

In conclusion, this paper presented an updated (to the year 2000) overview of the literature, addressing relevant issues of cast and direct post-and-core restorations. It provides useful information for dental clinicians, but it does not reveal high-level evidence. The question of whether cast post-and-cores perform better, equally or worse than direct post-and-core restorations remains to be answered. A systematic review requires more structure in its materials and methods.

\section{Practice point}

- The question of whether cast post-and-cores perform better, equally or worse than direct post-and-core restorations remains to be answered.

\section{Nico HJ Creugers}

Department of Occlusal Reconstruction and Oral Function, College of Dental Science, Faculty of Medical Sciences, University of Nijmegen, The Netherlands

1. Glenny AM, Esposito M, Coulthard P, Worthington HV. The assessment of systematic reviews in dentistry. Eur J Oral Sci 2003; 111:85-92.

2. Creugers NHJ, Mentink AGB, Käyser AF. An analysis of durability data on post and core restorations. J Dent 1993; 21:281-284.

3. Creugers NHJ, Kreulen CM. Systematic review of 10 years of systematic reviews in prosthodontics. Int I Prosthodont 2003: 16:123-127.

4. Reprinted from Journal of Prosthetic Dentistry, Vol 87, Heydecke G, Peters MC, The restoration of endodontically treated, single rooted teeth with cast or direct posts and cores: a systematic review, p. 380-386 (2002), with permission from the Editorial Council of the Journal of Prosthetic Dentistry.

Evidence-Based Dentistry (2003) 4, 89-90.

doi:10.1038/sj.ebd.6400191 